\title{
Timing of Tone Presentation Does Alter Training Performance but Not Retention Performance of a Point-to-Point Sequence Task
}

\author{
Arend W. A. Van Gemmert \\ School of Kinesiology, Louisiana State University, Baton Rouge, USA \\ Email: gemmert@1su.edu
}

Received June $1^{\text {st }}, 2012$; revised July $5^{\text {th }}, 2012$; accepted July $15^{\text {th }}, 2012$

\begin{abstract}
Objective: To investigate whether the timing of presentation of tones while practicing a serial reaction time task affects retention. Design: Thirty-eight young adults practiced 4 different 12 -sequence aiming tasks. There was one control condition without a tone and three experimental conditions in which a tone was presented; i.e., a tone could appear before the next target onset, at the next target onset, or after the next target onset. Sequence learning for each condition was assessed with a retention block in which no tones were presented. Performance changes as compared to the control condition were analyzed to assess if acquisition and/or learning was affected by the presentation of the tones. Results: Tone condition affected mainly reaction time. It was shown that if a tone was presented $150 \mathrm{~ms}$ before displaying the next target in the sequence the reaction time of the aiming movement decreased significantly. Furthermore, it was shown that tone onset $150 \mathrm{~ms}$ before target presentation and tone onset at target presentation resulted in a benefit during acquisition after block 5 and 6 respectively. However, the benefit disappeared during retention, because none of the tone conditions showed differential performance as compared to the control condition. Conclusions: Timing of tones affects the acquisition of serial reaction time tasks, but it does not alter learning. It is suggested that this pattern of findings supports the notion that tones result in a non-specific activation of the motor system, which affect immediate performance but not learning.
\end{abstract}

Keywords: Motor Learning; Activation; Sequence Learning; Serial Reaction Time (SRT) Task

\section{Introduction}

The serial response time (SRT) task (Nissen \& Bullemer, 1987 ) is the most used paradigm to investigate motor sequence learning (Song, Howard, \& Howard, 2008). It has been shown that during training, reaction times decrease faster when stimuli are repeated in a steady sequence than when stimuli are presented in random order (Nissen \& Bullemer, 1987). This phenomenon results in a steeper learning curve, which even can be found if subjects seemed to be unaware explicitly of the presence of a repeating sequence (Nissen \& Bullemer, 1987). The latter finding led to questions about the amount (and more recently the nature) of attentional resources needed for the occurrence of motor learning. These questions have led to several experiments involving dual task performance paradigms to investigate how allocation of attention to a secondary task would affect learning repeating sequences (Cohen, Ivry, \& Keele, 1990; McDowall, Lustig, \& Parkin, 1995; Schumacher \& Schwarb, 2009).

Researcher in a recent study noticed that, in support of earlier suggestions (e.g., Nissen \& Bullemer, 1987), learning of the sequence did not seem to depend on significant awareness of the pattern (Richard, Clegg, \& Seger, 2009). The recent study showed in addition that a sequence of direction without specific location was learned if the response required a movement into the required direction, while the sequence was not learned if the response did not involve a movement. One of the suggestions for the finding that direction was only learned when the required response includes a movement is that the execution of the movement results in focusing attention to the dimension(s) required to achieve the required goal (Richard et al., 2009). Thus, it seems that an important factor to learn a sequence is if attentional resources are devoted to learning the sequence.

A study that investigated the need for attentional resources while learning a sequence employed a dual task paradigm in which the secondary task was comprised of a tone pitch discrimination task in which the low pitch tones needed to be counted (McDowall et al., 1995). This study included a group of participants who were trained to perform the SRT task with the addition of secondary tone counting task, while another group of participants were trained without the secondary counting task. Although the secondary task did result in slower reaction times, it was shown that the sequence was learned with and without the secondary tone counting task. This study was comprised of four experiments and the first experiment showed that the only difference between the two groups was awareness of the sequence. When the participants learned the sequence without a secondary task $67 \%$ noticed that there was a sequence, even though only $11 \%$ were accurate in reproducing the entire 10 trial sequence. In contrast, when participants learned the sequence with the secondary task only $20 \%$ of the participants noticed a sequence and none of them were able to describe the entire sequence correctly. Thus, even though attentional resources are needed it does not necessary needs to be conscious. The other three experiments in this study basically confirmed the initial premise that awareness is not necessary to have (some) sequential learning effects in a SRT task. The latter notion that sequential learning effects occurs when a secondary task competes for resources needs some fine tuning, since it was shown that sequence learning is impeded when processes 
overlap in the dual task (Schumacher \& Schwarb, 2009).

Most studies investigating the effects of a secondary task on the acquisition of a SRT task have in common that the secondary task was comprised of some sort of tone counting and/or tone discrimination task in which the tones were presented during the inter stimulus/sequence interval. Furthermore, these studies emphasize the possible negative effects of these tones, while research has shown that the mere presentation of the tones could result in positive effects, most notably decreases in reaction time if presented before the go-stimulus, as result of a non-specific activation of the motor system (Van Gemmert \& Van Galen, 1994, 1997, 1998). In addition, the non-specific activation effects are assumed to be transient and to decay fast, so when the presentation of a tone occurs before the execution of the motor task, the negative effects as result of non-specific activation are expected to be minimal or gone at the time of onset of the movement. In contrast, when the presentation of the tones, resulting in non-specific activation of the motor system, occurs during the execution of a motor task with high accuracy demands, the non-specific activation results in an increase of noise in the system which negatively influences the motor performance (Van Gemmert \& Van Galen, 1998). Thus timing of tone presentation could have influenced the results of these studies. The latter suggestion that timing of the secondary task presentation is important together with the instruction given to participants has also been suggested by Schumacher and Schwarb (2009). They showed in their experiments and with an analysis of 21 studies that deterioration of SRT task performance occurs when the dual task requires higher demands due to instruction and/or timing of the presentation of the stimulus of the secondary task. Although, timing of the presentation of the stimulus for the secondary task is considered a factor for deteriorated performance, Schumacher and Schwarb (2009), like most researchers in this field, did not address the possibility that some deterioration could have been neutralized as result of the activational properties of a tone for the motor system (Van Gemmert \& Van Galen, 1994, 1997, 1998).

Another caveat of most studies is that the paradigm employed usually has limitations to reveal motor performance difficulties as it relates to the reaction and/or movement components of the performance, because these studies usually utilize a key press protocol which results in a motor response that is difficult to parse into separate movement components. Therefore, these studies are difficult to interpret as to location of its effects on the motor system beyond its general effects on the entire response. To investigate the possibility that the motor system is activated by tones, which possibly could lead to improved performance acquisition for some or all components of the response and maybe could result in (some) improvements in sequential learning, a variation on the classic SRT task was used in the current study. In this task participants had to make point-to-point movements with a stylus on a digitizer tablet allowing parsing responses in a reaction and movement component. Further more, to explore the effects of activation on the different components of the response three experimental conditions were administered in which the tones were presented either $150 \mathrm{~ms}$ before the appearance of the next target, at the same time the next target appeared, or $150 \mathrm{~ms}$ after the target had appeared. These conditions were contrasted to a control condition in which no tones were presented. The hypothesis investigated in the current study was that activation due to the presentation of a tone before each movement sequence improves ac- quisition of the SRT task, while activation during the execution of a sequence movement segment will adversely affect acquisition. The effects predicted during acquisition should remain during retention if acquisition is affected by the tones. Thus, as compared to acquisition in the control condition retention of the SRT task will show more efficient performance for sequences acquired with a tone presented before each movement segment, and the SRT task will show less efficient performance for sequences acquired with a tone presented during each movement segment.

\section{Method}

\section{Subjects}

Thirty-eight students of Louisiana State University between the ages of 20 and 30 years (Mean $=21.29 \pm 1.87$ years; 26 females, 12 males) participated in the experiment. All participants used their right-hand and they all reported to be right-hand dominant. Before participating, participants received an explanation of the experiment and they signed an informed-consent form. Participants filled out a short health history questionnaire, and anyone who indicated to have history of neurological problems, had current vision, or hearing problems, or were unable to hold and/or use a pen due to dexterity problems, were excluded from further participation. The protocol of the study was approved by the Human Subjects Institutional Review Board of Louisiana State University.

\section{Task and Design}

Participants were seated comfortably in a chair in front of a $50 \times 30 \mathrm{~cm}$ monitor and a digitizer tablet (WACOM Intuos 212 $\times 19$ ). The tablet recorded the $x-$ and $y$-position of an electronic pen with a sampling rate of $200 \mathrm{~Hz}$ and spatial resolution of $0.001 \mathrm{~cm}$. The experimental conditions were controlled by a program written in OASIS (KIKO Software, Doetinchem, The Netherlands). Participants Subjects were instructed to hold the pen using their normal pen grip and to draw lines from appearing circle to the next appearing circle as quickly and as accurately as possible. Target circles had a radius of $0.5 \mathrm{~cm}$ and did not disappear until the next target circle appeared which occurred $300 \mathrm{~ms}$ after the participants' pen arrived in the target circle. If the participant moved out of the target circle before the next target was presented, the next target would not appear and the participant was required to move the pen back in target circle. If this occurred this movement segment was deleted from the sequence. The distance between the targets was $7.5 \mathrm{~cm}$. During the experiment, the monitor provided visual feedback of the target circles and the on-line trajectory of the tip of the pen. A shield occluded vision of the participant's hand, forearm, and movement trajectory (see Figure 1).

There were three experimental practice conditions and one control practice condition. The order of conditions was counterbalanced according to a Latin-square design across participants. In the control and experimental conditions each trial consisted of a sequence of 12 targets in which 6 targets were located in the center of the screen (and digitizer) and 6 targets were located on the screen (and digitizer) at a $7.5 \mathrm{~cm}$ at one of the 12 outward positions at $0,30,60,90,120,150,180,210$, 240, 270, 300, and 330 degrees of the center target (see Figure 2). Each trial consisted of the same sequence of targets. However, each participant got a different sequence for each condition, 
in which 6 of the 12 outward targets each only appeared once. In an attempt to keep the complexity of each sequence the same only 24 predetermined sequences were used. To control for the possibility that one sequence would bias the results, each of the orders was at least used once in a particular tone condition across all participants, and none of the sequence orders was used more than twice in a particular tone condition across all participants. Before the experiment started participants were familiarized with the pen and with the point-to-point movement task by performing one trial of a movement sequence (without tones and headphones) which was not used in any of the conditions during the experiment.

The participants were only informed that 8 aiming sessions would be presented of which 4 of the sessions would be relatively long and 4 of the sessions would be relatively short. They were also informed that in some of the longer conditions it was possible that they would hear tones. No matter if they heard tones or not, they were instructed to concentrate on drawing a line ending in the target as fast and as accurate as possible. Thus, participants were not informed when tones would occur, and they were also not informed about the number of trials, when a trial started or ended, how many targets occurred in a trial, and the repetition of the same sequence in each trial.

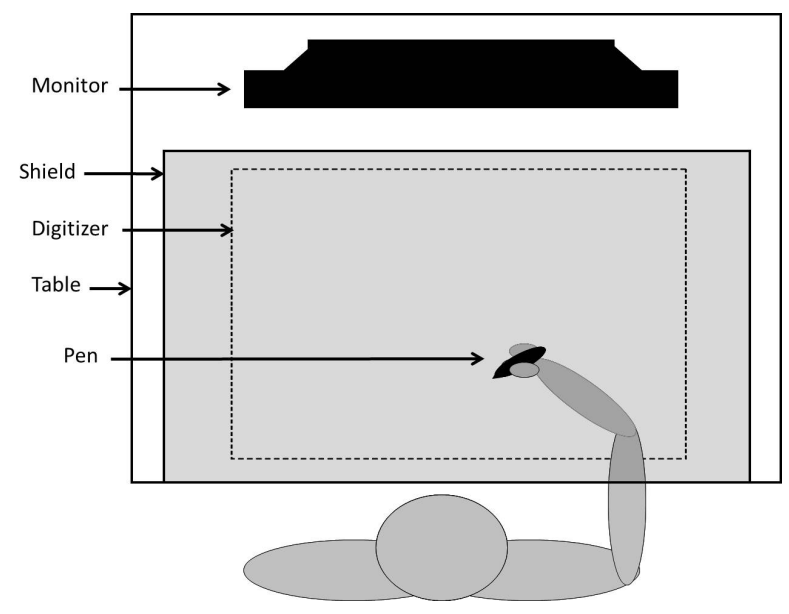

Figure 1.

Experimental setup.

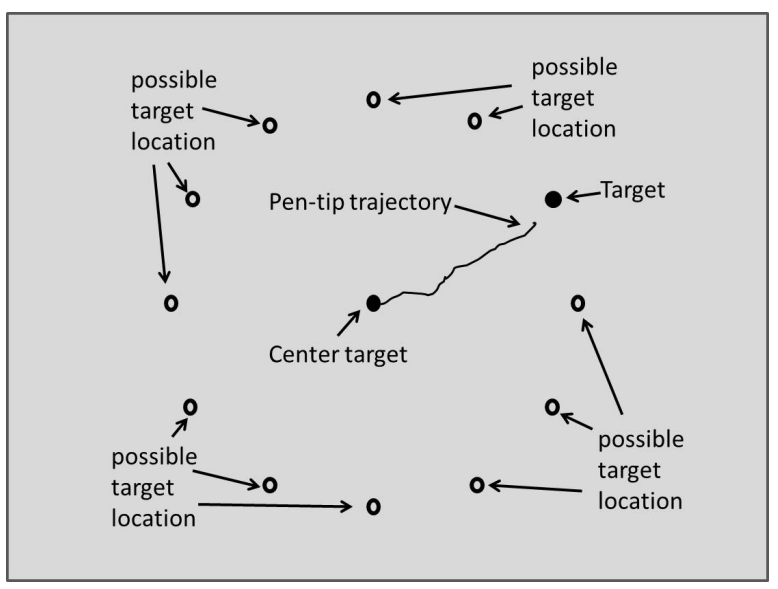

Figure 2.

Display of the task.
Participants received 40 practice trials and 4 retention trials 60 seconds after each practice session. Participants were required to wear headphones (Bose Quiet Comfort 15 Acoustic Noise Cancelling headphones, Bose Corporation, Farmingham, MA, USA) during the entire experiment. During the experimental practice trials an $80 \mathrm{~ms}$ tone was presented. In the three different experimental practice sessions the $80 \mathrm{~ms}$ tone was presented $150 \mathrm{~ms}$ before the target appeared ( -150 condition), at the same time when the target appeared ( 0 condition), or 150 ms after the target appeared ( +150 condition). No tones were presented during the control practice trials (No tone condition) and the retention trials.

\section{Data Analysis}

The recordings were processed with a custom program developed in OASIS (KIKO Software, Doetinchem, The Netherlands). The position signals were dual pass filtered with a Butterworth 4th order filter with a cutoff frequency of $7 \mathrm{~Hz}$. The onsets and offsets of pen tip movements were estimated by an algorithm that first located the $5 \%$ criterion of the peak in the absolute velocity profile. Then the algorithm went backwards from the $5 \%$ location to locate the onset to find the first location on the absolute velocity profile where absolute velocity was zero, stayed the same for $10 \mathrm{~ms}$, or was found to be the smallest in a period of $20 \mathrm{~ms}$. The same algorithm in reverse was used for movement offset. The dependent variables included duration of the whole sequence (DSeq), reaction time (RT), movement time (MT), number of velocity peaks (NVP), percentage of the duration of the total movement time spend in the primary sub-movement (PSMT) and accuracy of movement after the primary sub-movement (A-P-M). DSeq was defined as the time it took to make the 12 point-to-point movements. All other variables were determined per point-to-point movement. RT was defined as the time between appearance of the next target and the onset of the movement and MT was defined as the stroke duration between the onset and offset of the movement. NVP was determined by the number of local peaks in the tangential velocity profile; note that if a point-to-point movement is made in one smooth movement the tangential velocity profile will be bell-shaped with one single peak, if more than one peak occurs in the profile the movement is less smooth and assumed to be less automated (Meulenbroek \& Van Galen, 1988; Tucha, Mecklinger, Walitza, \& Lange, 2006). The primary sub-movement was defined as the distance between the location of the second zero crossing of the acceleration profile and the center of target (Ketcham, Seidler, Van Gemmert, \& Stelmach, 2002; Romero, Van Gemmert, Adler, Bekkering, \& Stelmach, 2003); note that if the velocity profile of the movement is perfectly bell-shaped with one single peak and no inflections, the primary sub-movement and the total movement are the same. To determine how efficiency of the movement was affected by condition and/or training PSMT and A-P-M were used. If the pointto-point movement shows a perfectly bell-shaped velocity profile with one single peak and no inflections, PSMT would be $100 \%$ and A-P-M would be $0 \mathrm{~mm}$. Thus, it is expected that when participants are learning the sequence they will get more efficient and thus PSMT should increase together with a decrease of A-P-M. Trials were divided in blocks of 4 and the average of the 4 trials were used to analyze effects of practice and retention resulting in 11 blocks in which the first 10 blocks were 
comprised of the 40 practice trials and the last 4 trials were comprised of the 4 retention trials. Thus repeated measures ANOVAs with two factors ( 4 conditions $\times 11$ blocks) were applied to all dependent variables. To determine practice and retention effects the first block was used as baseline performance, the 10th block was used as performance after training when tones were still present, and the 11th block was used to determine retention performance without tone present. Furthermore, if an interaction was found for blocks by tone conditions additional repeated measures one-way ANOVAs with the 4 tone conditions as factor per block were performed to determine which blocks showed significant effects of tone condition. For all significance levels of the ANOVAs Huyn-Feldt epsilon was used to adjust for possible violations of sphericity. If the ANOVAs showed significance bonferroni corrected t-tests with alpha set at 0.05 were applied to determine the locus of significance.

\section{Results}

\section{Sequence Duration}

It was shown that tone condition marginally affected sequence duration (DSeq), $\mathrm{F}[3,111]=2.277, p=0.085, \varepsilon=0.984)$. Blocks did significantly affect DSeq, F[10, 370] $=75.959, p<$ $0.001, \varepsilon=0.486$. Although inspection of Figure 3 suggests that some interaction may occur, the interaction between tone condition and blocks failed to reach significance for DSeq, $F[30$, $1110]=1.279, p=0.233, \varepsilon=0.371$.

Bonferroni corrected t-tests showed that DSeq reduced significantly from block 1 to 2 , and from 2 to 3 , after which reductions in DSeq became more modest. This resulted in the finding that blocks 1 and 2 differed significant from all other blocks, while block 3 did not differ significantly from block 4, 5 and 6 , it did significantly differ from all blocks after the 6th block. A final finding was that DSeq of block 7 and 9 was significantly smaller than DSeq of all blocks previous to block 6 (see Figure 3). This latter finding that block 7 and 9 differed significantly from blocks 4 and 5 in addition to the blocks 1, 2 and 3 may have been caused by the decrease in DSeq of the $-150 \mathrm{~ms}$ condition for these blocks even though an interaction between blocks and tone condition did not reach significance (see Figure 3).

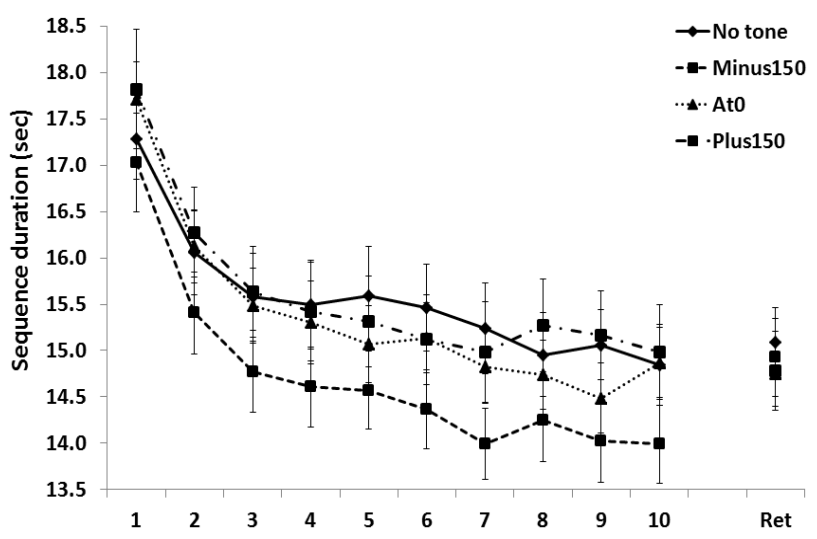

Figure 3.

Sequence duration as function of tone condition and blocks (Ret $=$ retention block; No tone $=$ no tones were presented; Minus $150=$ tone 150 $\mathrm{ms}$ before target presentation; At $0=$ tone when target is presented; Plus $150=$ tone $150 \mathrm{~ms}$ after target presentation).

\section{Reaction Time}

Reaction time (RT) was significantly affected by tone condition $(\mathrm{F}[3,111]=5.279, p=0.002, \varepsilon=0.981)$, and blocks, $\mathrm{F}[10$, $370]=83.159, p<0.001, \varepsilon=0.480$. The interaction of tone condition and blocks on RT approached significance, $\mathrm{F}[30$, $1110]=1.732, p=0.054, \varepsilon=0.417$ (see Figure 4).

Follow-up analysis showed that RT was significantly smaller when the tone occurred $150 \mathrm{~ms}$ before the target was displayed (i.e., $-150 \mathrm{~ms}$ tone condition) as compared to the condition where the tone occurred $150 \mathrm{~ms}$ after target presentation (i.e., $150 \mathrm{~ms}$ tone condition) or when no tone occurred (i.e., control condition).

Similarly to findings for effects of blocks for DSeq, bonferroni corrected t-tests showed that RT reduced significantly from block 1 to 2 and from block 2 to 3 , after which reductions of RT became more moderate, resulting in the finding that blocks 1 and 2 differed significant from all other blocks, while the reduction in RT from block 3 to 4 was not significant, but was significant when block 3 was compared to $6,7,8,9$, and 10 . Again RT in block 7 and 9 was significant smaller than RT of all block previous to the 6th block. In contrast to DSeq, RT of the retention block was significantly larger than block 7 and 9 , even though it was significantly smaller than the RT in block 1 and 2.

Analysis per block showed that the interaction of tone condition by block was caused by a gradual separation of the -150 $\mathrm{ms}$ and $0 \mathrm{~ms}$ tone conditions from the $150 \mathrm{~ms}$ and control tone condition during training which all again united in the retention block. In particular RT started to be significantly smaller than $\mathrm{RT}$ of $150 \mathrm{~ms}$ and the control condition in block 5 for the -150 $\mathrm{ms}$ tone condition and RT of the $0 \mathrm{~ms}$ tone condition started to become smaller in block 6 (see Figure 4).

\section{Movement Time}

Movement time (MT) was not affected by tone condition, $\mathrm{F}[3,111]=0.644, p=0.579, \varepsilon=0.871$. The main effect of blocks on MT proved to be significant, $\mathrm{F}[10,370]=30.103, p$ $<0.001, \varepsilon=0.489$. The interaction of tone condition and blocks on MT did not show a significant effect, $\mathrm{F}[30,1110]=0.904, p$ $=0.517, \varepsilon=0.280$.

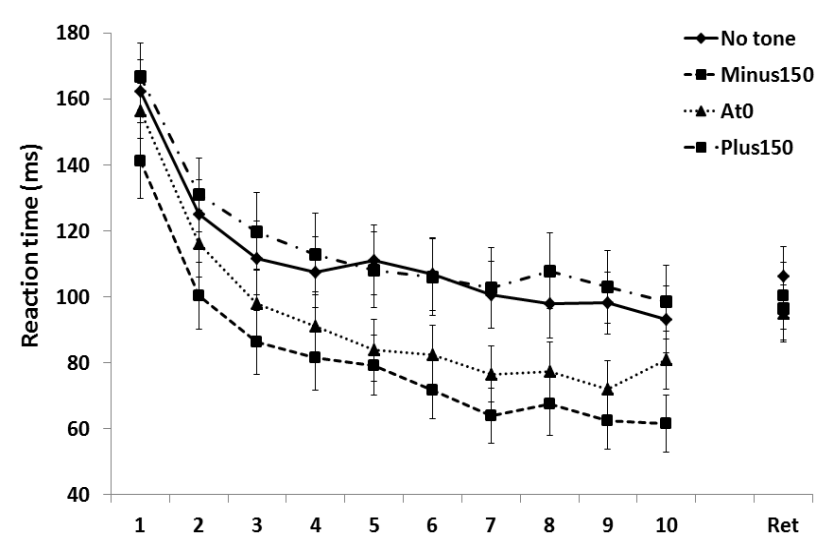

Figure 4.

Reaction time as function of tone condition and blocks $($ Ret $=$ retention block; No tone $=$ no tones were presented; Minus $150=$ tone $150 \mathrm{~ms}$ before target presentation; At $0=$ tone when target is presented; Plus $150=$ tone $150 \mathrm{~ms}$ after target presentation). 
Bonferroni corrected t-tests showed that MT was significantly reduced from block 1 to block 2 , while the reductions on average after block 2 did not differ significant from each consecutive block. The small reductions of MT after block 2 lead finally to a significant smaller MT in block 11 (the retention block) as compared to block 2 (see Figure 5).

\section{Number of Velocity Peaks}

The pattern of results for number of velocity peaks (NVP) mirrored those of the MT, NVP was not affected by tone condition, $\mathrm{F}[3,111]=1.474, p=0.226, \varepsilon=1.0$. The main effect of block on NVP proved to be significant, $\mathrm{F}[10,370]=13.330, p$ $<0.001, \varepsilon=0.455$. And the interaction of tone condition and block on NVP did not reach significance, $F[30,1110]=0.781$, $p=0.677, \varepsilon=0.420$.

Again a similar pattern of results as found for MT emerged for NVP. Bonferroni corrected t-tests showed that NVP was significantly reduced from block 1 to block 2 , while the reductions on average after block 2 did not differ significant from each consecutive block. The small reductions of NVP after block 2 lead finally to a significant smaller NVP in block 11 (the retention block) as compared to block 2 (see Figure 6).

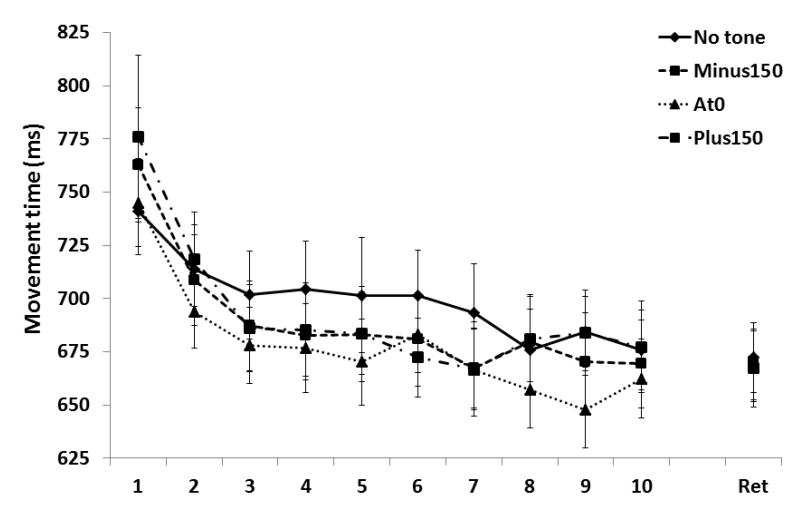

Figure 5.

Movement time as function of tone condition and blocks (Ret $=$ retention block; No tone $=$ no tones were presented; Minus $150=$ tone $150 \mathrm{~ms}$ before target presentation; At $0=$ tone when target is presented; Plus $150=$ tone $150 \mathrm{~ms}$ after target presentation).

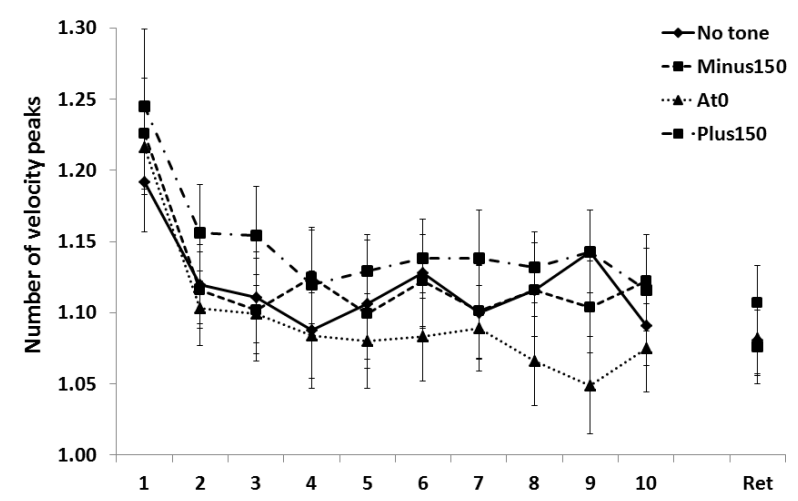

Figure 6.

Number of velocity peaks as function of tone condition and blocks $($ Ret $=$ retention block; No tone $=$ no tones were presented; Minus $150=$ tone $150 \mathrm{~ms}$ before target presentation; At $0=$ tone when target is presented; Plus150 = tone $150 \mathrm{~ms}$ after target presentation).

\section{Sub-Movement Measures}

The percentage of the duration of the total movement time spend in the primary sub-movement (PSMT) and the accuracy of movement after the primary sub-movement (A-P-M) were not changed as result of tone condition, $\mathrm{F}[3,111]=1.588, p=$ $0.206, \varepsilon=0.803$, and $\mathrm{F}[3,111]=1.947, p=0.148, \varepsilon=0.695$, respectively. Whereas the main effect of block on PSMT did not show significance $(\mathrm{F}[10,370]=1.612, p=0.126, \varepsilon=$ 0.756 ), the factor block showed a significant effect on A-P-M, $\mathrm{F}[10,370]=1.928, p=0.050, \varepsilon=0.868$. The interaction of tone condition and block on both PSMT and A-P-M proved to be marginally significant, $\mathrm{F}[30,1110]=1.542, p=0.054, \varepsilon=$ 0.728 , and $\mathrm{F}[30,1110]=1.449, p=0.087, \varepsilon=0.709$.

Even though A-P-M showed a significant effect of blocks and a marginal significant interaction of tone condition and blocks, the post-hoc analysis did not reveal that any of the blocks and/or tone conditions differed significantly from each other. In fact the largest difference on average between two blocks was $0.80 \mathrm{~mm}$ (block $1=10.87 \mathrm{~mm}$; retention block = $10.07 \mathrm{~mm}$ ) and if changes over blocks in A-P-M were taken for each tone condition separate than the no tone condition showed an improvement from block 1 to the retention block of $0.97 \mathrm{~mm}$. Even if tone conditions were compared per block the largest difference was found to be pretty small, because the largest difference found was $1.67 \mathrm{~mm}$ between the +150 condition in block $6(9.53 \mathrm{~mm})$ and the no tone condition in the same block $(11.20 \mathrm{~mm})$. Also PSMT did not show any large differences, whereas the average improvement from block $1(88.45 \%)$ to the retention block $(89.84 \%)$ was only $1.39 \%$, the largest difference was found in the -150 condition where the retention block (89.79\%) improved $1.82 \%$ compared to block 1 (87.97\%). In summary, the kinematic structure of the sub-movements cannot explain the learning and/or tone presentation effects found in the overall measures.

\section{Discussion}

It was shown that tones, when presented before sequence segments in a SRT task, resulted in a trend that showed shorter overall sequence durations during acquisition. However, the small benefits of tones observed during acquisition trials did not result in learning benefits. Moreover, the benefits disappeared during retention trials when tones were not presented and the learned sequence showed similar retention performance as the control condition without tones. Thus, it seems that the addition of tones to a motor sequence learning protocol does not alter learning. This latter finding is perhaps important to note, since it means that the tones did not improve or hamper learning, even though during acquisition they did benefit performance.

Traditionally tone presentation during the acquisition of a motor sequence has been used to investigate attentional resources (Bullemer \& Nissen, 1990; McDowall et al., 1995; Nissen \& Bullemer, 1987). However in the current study, participants were instructed to concentrate on drawing a line ending in the target as fast and as accurate as possible and they were told that should focus on the motor task at hand no matter if they heard tones or not. Therefore this study cannot be interpreted as to its effects on attentional resources from a dual-task perspective. Nevertheless, attention could have played a role. It is feasible to envision that a tone occurring before execution of the movement sequence will act as a warning stimulus, while a tone 
occurring at the same time as when the next target appears will improve detection of the go-stimulus. Both of these tone conditions could result in narrowing the focus of attention on the point-to-point aiming task which means that all available attentional resources would be directed to the most optimal performance of the task. Furthermore, when the tone is presented during execution it could act as a distraction resulting in diminishing some of the resources used to execute the point-to-point aiming task. When compared to the condition when no tones were presented during acquisition, these assumptions about tones affecting attentional resource allocation should have resulted into improved retention performance for the two tone conditions and deteriorated performance for the condition in which a tone was presented during execution of the aiming movement. Moreover, this prediction for the pattern of retention is based on the view that when more attentional resources are dedicated to the acquisition of a task, the task is learned better and thus performance during retention should be better than retention performance when less attentional resources were dedicated to the task during training. In the current study this pattern of retention performance was not found, because retention performance was the same for all conditions, therefore it is deemed to be unlikely that the tone presentation in the current study altered normal management of attentional resources. Off course, it should be noted that the current study did not include conditions in which the participants were instructed to attend to the tones so one cannot make strong arguments about the use of attentional resources and its effects on task performance.

In depth analyses, in which each movement sequence was parsed into a reaction and execution portion, showed that reaction time decreased, in addition to the normal reductions observed during acquisition, when tones were presented before and at the same time as the presentation of the movement target. Nevertheless, these benefits for reaction time during acquisition did not translate to additional benefits for retention performance when compared to retention performance without tones.

Whereas reaction time was affected by tones during acquisition, movement speed, smoothness, and execution efficiency were not altered by the tones as indicated by the findings that tone condition did not show main effects for movement time, number of velocity peaks or any of the sub-movement time measures. Therefore, it can be concluded that only transient preparatory processes which are only involved in movement initiation related activities benefit of the tones. When assumed that the task is easy or becomes very fast easy with practice, the results that the tones benefit preparatory processes is in line with the theoretical perspective that tones increase non-specific activation in the motor system, which benefits simple aiming tasks (Van Gemmert \& Van Galen, 1998). Another possibility for improvements in reaction time during acquisition is that the organization of the trials was very consistent, and it has been suggested that learning depends on practicing run of trials that are consistently organized (Stadler, 1995). However, this explanation has difficulty to explain the finding that improvements in reaction time do disappear during retention when the trials do not include a tone. Moreover, since the trials during acquisition include tones and the trials during retention do not include tones, it is expected that reaction time would be worse than retention for the no tone condition, because the latter condition has consistency of organization across acquisition and retention, while the experimental conditions are not consistent in organization across acquisition and retention.

In summary, the current study showed evidence that tones affect acquisition performance depending on the time of presentation, however, it does not alter learning. More specifically, the tones prove to be performance variables that do not alter the execution phase of performance, but it has a direct impact on reaction time. This pattern together with the finding that tones do not alter learning supports the hypothesis that these tones result in a non-specific activation of the motor system (Van Gemmert \& Van Galen, 1997).

\section{REFERENCES}

Bullemer, P., \& Nissen, M. J. (1990). Attentional orienting in the expression of procedural knowledge. Bulletin of the Psychonomic Society, 28, 505-505.

Cohen, A., Ivry, R. I., \& Keele, S. W. (1990). Attention and structure in sequence learning. Journal of Experimental Psychology-Learning Memory and Cognition, 16, 17-30. doi:10.1037/0278-7393.16.1.17

Ketcham, C. J., Seidler, R. D., Van Gemmert, A. W., \& Stelmach, G. E. (2002). Age-related kinematic differences as influenced by task difficulty, targetsize, and movement amplitude. The Journals of Gerontology: Series B, 57, 54-64. doi:10.1093/geronb/57.1.P54

McDowall, J., Lustig, A., \& Parkin, G. (1995). Indirect learning of event sequences: The effects of divided attention and stimulus continuity. Canadian Journal of Experimental Psychology-Revue Canadienne De Psychologie Experimentale, 49, 415-436. doi:10.1037/1196-1961.49.4.415

Meulenbroek, R. G., \& Van Galen, G. P. (1988). Foreperiod duration and the analysis of motor stages in a line-drawing task. Acta Psychologica, 69, 19-34. doi:10.1016/0001-6918(88)90027-3

Nissen, M. J., \& Bullemer, P. (1987). Attentional requirements of learning-Evidence from performance-measures. Cognitive Psychology, 19, 1-32. doi:10.1016/0010-0285(87)90002-8

Richard, M. V., Clegg, B. A., \& Seger, C. A. (2009). Implicit motor sequence learning is not represented purely in response locations. Quarterly Journal of Experimental Psychology, 62, 1516-1522. doi:10.1080/17470210902732130

Romero, D. H., Van Gemmert, A. W., Adler, C. H., Bekkering, H., \& Stelmach, G. E. (2003). Time delays prior to movement alter the drawing kinematics of elderly adults. Human Movement Science, 22, 207-220. doi:10.1016/S0167-9457(02)00160-4

Schumacher, E. H., \& Schwarb, H. (2009). Parallel response selection disrupts sequence learning under dual-task conditions. Journal of Experimental Psychology-General, 138, 270-290. doi: $10.1037 / \mathrm{a} 0015378$

Song, S., Howard, J. H., \& Howard, D. V. (2008). Perceptual sequence learning in a serial reaction time task. Experimental Brain Research, 189, 145-158. doi:10.1007/s00221-008-1411-z

Stadler, M. A. (1995). Role of attention implicit learning. Journal of Experimental Psychology-Learning Memory and Cognition, 2, 674685. doi: $10.1037 / 0278-7393.21 .3 .674$

Tucha, O., Mecklinger, L., Walitza, S., \& Lange, K. W. (2006). Attention and movement execution during handwriting. Human Movement Science, 25, 536-552. doi:10.1016/j.humov.2006.06.002

Van Gemmert, A. W., \& Van Galen, G. P. (1994). Effects of a seconddary, auditory task on graphic aiming movements. In C. Faure, P. Keuss, G. Lorette, \& A. Vinter (Eds.), Advances in handwriting and drawing: A multidisciplinary approach (pp. 421-439). Paris: Europia.

Van Gemmert, A. W., \& Van Galen, G. P. (1997). Stress, neuromotor noise, and human performance: A theoretical perspective. The Journal of Experimental Psychology: Human Perception and Performance, 23, 1299-1313. doi:10.1037/0096-1523.23.5.1299

Van Gemmert, A. W., \& Van Galen, G. P. (1998). Auditory stress effects on preparation and execution of graphical aiming: A test of the neuromotor noise concept. Acta Psychologica, 98, 81-101. doi:10.1016/S0001-6918(97)00049-8 